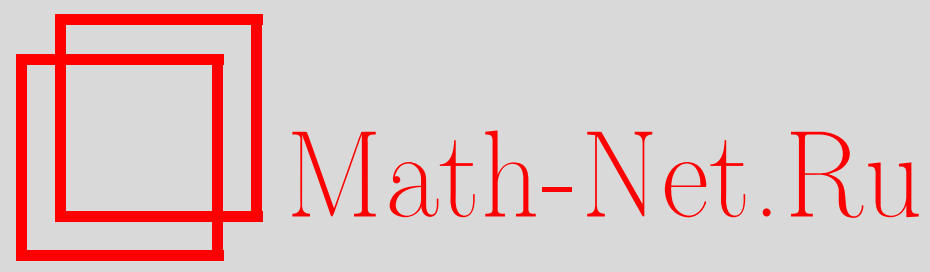

Г. А. Омельянов, В. В. Трушков, Геометрическая поправка в задаче о движении свободной границы, Матем. заметки, 1998, том 63, выпуск 1, 151-153

DOI: https://doi.org/10.4213/mzm1261

Использование Общероссийского математического портала Math-Net.Ru подразумевает, что вы прочитали и согласны с пользовательским соглашением http://www.mathnet.ru/rus/agreement

Параметры загрузки:

IP: 35.174 .16 .151

26 апреля 2023 г., 08:00:28

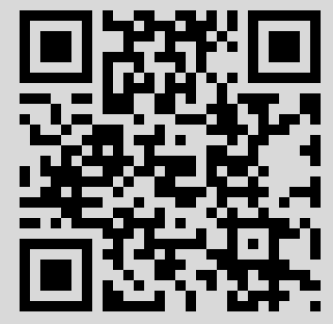




\section{ГЕОМЕТРИЧЕСКАЯ ПОПРАВКА \\ В ЗАДАЧЕ О ДВИЖЕНИИ СВОБОДНОЙ ГРАНИЦЫ}

\section{Г. А. Омельянов, В.В. Трушков} ля [1]

В работе построена поправка к асимптотическому решению системы уравнений фазового по-

$$
\begin{gathered}
\partial_{t}(\theta+\varphi)=\Delta \theta, \quad t \in(0, T), \quad x \in \Omega \\
\varepsilon^{2} \partial_{t} \varphi=\varepsilon^{2} \Delta \varphi+\varphi-\varphi^{3}+\varepsilon \varkappa \theta \\
\left.\theta\right|_{\Sigma}=\left.g(x, t)\right|_{\Sigma},\left.\quad \varphi\right|_{\Sigma}=1,\left.\quad \theta\right|_{t=0}=\theta_{0}(x, \varepsilon),\left.\quad \varphi\right|_{t=0}=\varphi_{0}(x, \varepsilon) .
\end{gathered}
$$

Здесь $\Sigma=\partial \Omega \times(0, T), \Omega$ - область в $\mathbb{R}^{n}$ с гладкой $\left(C^{\infty}\right)$ границей, $\varepsilon$ - малый параметр, $\varkappa>0$ константа, $\partial_{t}=\partial / \partial t$.

Предполагается, что существуют подобласти $\Omega_{0}^{ \pm}$такие, что $\Omega=\Omega_{0}^{+} \cup \Omega_{0}^{-} \cup \Gamma_{0}, \Gamma_{0}-$ замкнутое гладкое многообразие коразмерности 1 , не имеющее общих точек с внешней границей $\partial \Omega$, и что при $\varepsilon \rightarrow 0$ и $x \in \Omega_{0}^{ \pm}, \theta_{0}(x, \varepsilon) \rightarrow \theta_{0}^{ \pm}(x) \in C^{\infty}\left(\Omega_{0}^{ \pm}\right), \varphi_{0}(x, \varepsilon) \rightarrow \pm 1$.

$\mathrm{B}$ [2] на базе результатов [3], [4] разработана схема построения самоподобной асимптотики типа кинка для задачи (1), (2). Показано, что построение главного члена сводится к решению модифицированной задачи Стефана (см. также [1])

$$
\begin{gathered}
\partial_{t} \theta^{ \pm}-\Delta \theta^{ \pm}=0, \quad x \in \Omega_{t}^{ \pm}, \quad t \in(0, T), \\
\left.\theta^{ \pm}\right|_{t=0}=\theta_{0}^{ \pm}, \quad x \in \Omega_{0}^{ \pm},\left.\quad \theta^{+}\right|_{\Sigma}=\left.g(x, t)\right|_{\Sigma}, \\
{\left.\left[\theta^{ \pm}\right]\right|_{\Gamma_{t}}=0,\left.\quad\left[\partial_{\nu} \theta^{ \pm}\right]\right|_{\Gamma_{t}}=2 V_{\nu},} \\
\left.\theta^{+}\right|_{\Gamma_{t}}=\varkappa_{1} K_{t}-\varkappa_{1} V_{\nu} .
\end{gathered}
$$

Здесь $\Omega_{t}^{ \pm}$- подобласти $\Omega$ такие, что $\Omega=\Omega_{t}^{+} \cup \Omega_{t}^{-} \cup \Gamma_{t}, \Gamma_{t}$ обладает теми же свойствами, что и $\Gamma_{0}, V_{\nu}$ - нормальная скорость движения $\Gamma_{t}, K_{t}-$ средняя кривизна $\Gamma_{t}, \varkappa_{1}=\sqrt{2} /(3 \varkappa)$, через $[f]$ обозначен скачок $f$ на $\Gamma_{t}, \partial_{\nu}=\partial / \partial_{\nu}$ - производная вдоль нормали к $\Gamma_{t}$.

Условие (6) называется условием Гиббса-Томсона. Оно является частным случаем известной задачи о средней кривизне, т.е. задачи определения свободной поверхности, подчиняющейся соотношению $V_{\nu}=K_{t}+f$.

Положение фронта в задаче (3)-(6) описывается с помощью вспомогательной функции $\psi(x)$, т.е. $\Gamma_{t}=\{x \in \Omega, t=\psi(x)\}$; нормальная скорость и кривизна вычисляются по формулам $V_{\nu}=-1 /|\psi(x)|$, $K_{t}=\operatorname{div} \nu$, где $\nu=\nabla \psi(x) /|\nabla \psi(x)|-$ нормаль $\mathrm{\kappa} \Gamma_{t}, \partial_{\nu}=\langle\nu, \nabla\rangle$. Известно, что при неособой поверхности с ненулевой средней кривизной условие Гиббса-Томсона (6) переписьвается в виде квазилинейного параболического уравнения (дополненного начальным условием $\left.\psi\right|_{\Gamma_{0}}=0$ ), в котором роль времени играет параметр вдоль нормали к $\Gamma_{t}[2]$.

Таким образом, в главном члене определение фронта кинка сводится к задаче о средней кривизне. Однако, существуют задачи, в которых необходимо знать поправки (по крайней мере величины $O(\varepsilon))$ к закону движения свободной границы. Пример - задача о так называемых волновых поездах, в которой переходная зона моделируется суперпозицией $M$ кинков $\left(M=O\left(1 / \varepsilon^{\gamma}\right), \gamma \in(0,1)\right)$, фронты которых расположены на расстоянии $O\left(\varepsilon^{\gamma}\right)$ друг от друга [5], [6]. Ясно, что в этой задаче необходимо описывать положение фронта с точностью большей, чем $O(1)$.

Работа выполнена при частичной поддержке Российского фонда фундаментальных исследований, грант № 96-01-10492. 
Сформулируем основной результат работы. Для этого определим функции $\theta_{1}^{ \pm}=\theta_{1}^{ \pm}(x, t)$ и $s=s(x)$ как решение задачи

$$
\begin{gathered}
\partial_{t} \theta_{1}^{ \pm}=\Delta \theta_{1}^{ \pm}-\frac{\varkappa}{2} \partial_{t} \theta^{ \pm}, \quad x \in \Omega_{t}^{ \pm}, \quad t \in(0, T), \\
\left.\theta_{1}^{ \pm}\right|_{t=0}=\theta_{ \pm}^{1}(x), \quad x \in \Omega_{0}^{ \pm},\left.\quad \theta_{1}^{+}\right|_{\Sigma}=0, \\
{\left.\left[\theta_{1}^{ \pm}\right]\right|_{\Gamma_{t}}=0,\left.\quad\left[\partial_{\nu} \theta_{1}^{ \pm}\right]\right|_{\Gamma_{t}}=-p_{1} V_{\nu},} \\
\varkappa_{1}\left(\widehat{\mathscr{K}}_{t}^{\prime}-\widehat{V}_{\nu}^{\prime}\right) s=\left.\left(\theta_{t}^{+}+\theta_{t}^{-}\right)\right|_{\Gamma_{t}} \frac{s}{2}-\frac{|\nabla \psi|}{\sqrt{2}}-\left.2 \theta_{1}^{+}\right|_{\Gamma_{t}},
\end{gathered}
$$

где

$$
p_{1}=\frac{1}{2}(\Delta \psi+1) s\left(|\nabla \psi|^{2}+\left.|\nabla \psi|\left[\partial_{\nu} \theta_{t}^{ \pm}\right]\right|_{\Gamma_{t}}\right), \quad \theta_{ \pm}^{1}=\lim _{\varepsilon \rightarrow 0} \frac{\theta_{0}-\theta_{0}^{ \pm}}{\varepsilon}
$$

$\left(\widehat{\mathscr{K}}_{t}^{\prime}-\widehat{V}_{\nu}^{\prime}\right) s-$ вариация $\mathscr{K}_{t}-V_{\nu}$, которая действует по формуле

$$
\left(\widehat{\mathscr{K}}_{t}^{\prime}-\widehat{V}_{\nu}^{\prime}\right) s=\left\langle\nabla, \frac{\nabla s-\langle\nu, \nabla s\rangle \nu}{|\nabla \psi|}\right\rangle-\frac{\langle\nu, \nabla s\rangle}{|\nabla \psi|}
$$

Обозначим через $\widetilde{\theta}_{0}^{ \pm}(x, t)$ (соответственно $\left.\widetilde{\theta}_{1}^{ \pm}(x, t)\right)$ гладкие продолжения $\theta^{ \pm}\left(\theta_{1}^{ \pm}\right)$в области $\Omega_{t}^{\mp}$ такие, что каждая из функций $\widetilde{\theta}_{0}^{ \pm}\left(\widetilde{\theta}_{1}^{ \pm}\right)$удовлетворяет в $\Omega_{t}^{\mp}$ уравнению теплопроводности (неоднородному уравнению с правой частью $\left.\widetilde{f}_{1}^{ \pm}=-\frac{\varkappa}{2}\left(\partial \widetilde{\theta}^{ \pm} / \partial t\right)\right)$.

Tеорема. Асимптотическое по $\bmod O\left(\varepsilon^{2}\right)$ решение типа кинка системы уравнений фазового поля имеет вид

$$
\begin{gathered}
\varphi=\operatorname{th}(\beta \tau)+\varepsilon\left(\frac{\varkappa}{4}\left(\widetilde{\theta}_{0}^{+}+\widetilde{\theta}_{0}^{-}\right)+\frac{\varkappa}{4}\left(\widetilde{\theta}_{0}^{+}-\widetilde{\theta}_{0}^{-}\right) \chi+\left(\frac{1}{\sqrt{2}}\left(\partial_{\nu} \beta\right) \tau^{2}+\sqrt{2} \beta\left(\partial_{\nu} s\right) \tau+c\right) \chi_{\tau}\right), \\
\theta=\frac{1}{2}\left(\widetilde{\theta}_{0}^{+}+\widetilde{\theta}_{0}^{-}\right)+\frac{1}{2}\left(\widetilde{\theta}_{0}^{+}-\widetilde{\theta}_{0}^{-}\right) \chi \\
\quad+\varepsilon\left(\frac{1}{2}\left(\widetilde{\theta}_{1}^{+}+\widetilde{\theta}_{1}^{-}\right)+\left(\frac{1}{2}\left(\widetilde{\theta}_{1}^{+}-\widetilde{\theta}_{1}^{-}\right)\right) \chi-2 \beta \varkappa(\beta \tau-\ln (2 \operatorname{ch}(\beta \tau)))\right) .
\end{gathered}
$$

Здесь $\chi=\operatorname{th}(\beta \tau), \tau=(t-\psi(x)+\varepsilon s(x)) / \varepsilon, \beta=|\nabla \psi| / \sqrt{2}, c=c(x)-$ постоянная интедрирования, которая определяется при построении членов асимптотического решения величины $O\left(\varepsilon^{2}\right)$.

Как следует из теоремы, положение фронта кинка с точностью $O\left(\varepsilon^{2}\right)$ определяется равенством $t=\psi(x)-\varepsilon s(x)$. В свою очередь, поправка $s$ находится из линеаризованной модифиццированной задачи Стефана (7)-(10). Можно доказать, что при достаточно малых $T$ (но не зависящих от $\varepsilon$ ) задача (7)-(10) имеет классическое решение при тех же предположениях, что и задача (3)-(6) (см. [2]).

Наметим доказательство теоремы. В [2] показано, что для уравнений (1) асимптотика типа кинка представима в виде

$$
\begin{gathered}
\theta(x, t, \varepsilon)=\Xi^{N}(x, t, \varepsilon)+V^{N}\left(\frac{S}{\varepsilon}, x, t, \varepsilon\right)+O\left(\varepsilon^{N+1}\right), \\
\varphi(x, t, \varepsilon)=W^{N}\left(\frac{S}{\varepsilon}, x, t, \varepsilon\right)+\varepsilon \Phi^{N}(x, t, \varepsilon)+O\left(\varepsilon^{N+1}\right),
\end{gathered}
$$


где

$$
\begin{gathered}
V^{N}(\tau, x, t, \varepsilon)=\rho(x, t) V_{0}(\tau, x, t)+\sum_{j=1}^{N}\left(\rho_{j}(x, t) V_{j}(\tau, x, t)+U_{j}(\tau, x, t)\right), \\
W^{N}(\tau, x, t, \varepsilon)=\chi(\tau, x)+\sum_{j=1}^{N} \varepsilon^{j} W_{j}(\tau, x, t), \\
\Theta^{N}(x, t, \varepsilon)=\sum_{j=0}^{N} \varepsilon^{j} \theta_{j}(x, t), \quad \Phi^{N}(x, t, \varepsilon)=\sum_{j=1}^{N} \varepsilon^{j-1} \varphi_{j}(x, t) .
\end{gathered}
$$

Здесь $S=t-\psi(x)+\varepsilon s(x) ; \psi, s \in C^{\infty}(\bar{\Omega}) ; \theta_{j}, \varphi_{j}, \rho_{j} \in C^{\infty}(\bar{Q}), Q=\Omega \times(0, T)$, причем $\left.\rho_{j}\right|_{t=\psi(x)}=0 ; V_{j}(\tau, x, t), U_{j}(\tau, x, t), W_{j}(\tau, x, t), \chi(\tau, x) \in \mathscr{H}$, и мы используем обозначения $\mathscr{H}=\left\{f: f_{\tau}^{\prime} \in \mathscr{S}\right\}$, где $\mathscr{S}=\left\{f: f(\tau, x, t) \in S\left(\mathbb{R}^{1} ; C^{\infty}(Q)\right)\right\}, S$ - пространство Шварца. Подставив разложения (11) в (1), приравняв нулю члены одного порядка по $\varepsilon$ и устремив $\tau$ к $\pm \infty$, получаем соотношения между

$$
\varphi_{j}^{ \pm}=\lim _{\tau \rightarrow \pm \infty}\left(\varphi_{j}+W_{j}\right) \text { и } \theta_{j}^{ \pm}=\lim _{\tau \rightarrow \pm \infty}\left(\theta_{j}+\rho_{j} V_{j}+U_{j}\right), \quad j \geqslant 0
$$

в частности, равенство $\varphi_{1}^{ \pm}=\varkappa \theta_{0}^{ \pm} / 2$, а также уравнения

$$
\left(\partial_{t}-\Delta\right) \theta_{0}^{ \pm}=0, \quad\left(\partial_{t}-\Delta\right) \theta_{j}^{ \pm}=-\partial_{t} \varphi_{j}^{ \pm}, \quad j=1, \ldots, N-1 .
$$

Уравнения (12) при $j=1$ приводят к уравнениям теплопроводности (3) и (7).

Построение быстро меняющихся членов асимптотики сводится к решению обыкновенных уравнений

$$
\begin{gathered}
|\nabla \psi|^{2} \chi_{\tau \tau}+\chi-\chi^{3}=0 \\
|\nabla \psi|^{2} W_{j \tau \tau}+\left(1-3 \chi^{2}\right) W_{j}=F_{j}^{\varphi}(\tau, x, t), \quad U_{j \tau \tau}=F_{j}^{\theta}(\tau, x, t) .
\end{gathered}
$$

Из (13) мы находим $\chi=\operatorname{th}(\beta \tau)$, а условия разрешимости (14) в $\mathscr{H}$ приводят к дополнительным уравнениям на $\psi, s$ и "постоянным" интегрирования (14). В частности, эти условия при $j=1$ приводят к условиям Стефана (5) и Гиббса-Томсона (6), а при $j=2$-к линеаризованным условиям Стефана (9) и Гиббса-Томсона (10) для первой поправки.

\section{СПИСОК ЦИТИРОВАННОЙ ЛИТЕРАТУРЫ}

1. Caginalp G. // Arch. Rational Mech. Anal. 1986. V. 92. P. 205-245. 2. Данилов В. Г., Омельянов Г. А., Радкевич Е. В. // Дифференц. уравнения. 1995. Т. 31. № 3. С. 483-491. 3. Маслов В. П., Омельянов Г. А. // УМН. 1981. Т. 36. № 3. С. 63-126. 4. Маслов В. П., Данилов В. Г., Волосов К. А. Математическое моделирование процессов тепломассопереноca. M.: Наука, 1987. 5. Lacey A. A., Taylor A. B. // IMA J. Appl. Math. 1983. V. 30. P. 303-314. 6. Danilov V. G., Omel'yanov G. A., Radkevich E. V. // Free Boundary Problem News. 1995. V. 6. P. 27-28. 